\title{
Effect of Cognitive-Linguistic Intervention in Older Adults
}

\author{
Mi Sook Lee \\ Department of Audiology \& Speech-Language Pathology, Hallym University of Graduate Studies, Seoul, Korea \\ 노인에 대한 인지-언어적 중재 효과
}

이 미 숙

한림국제대학원대학교 청각언어치료학과

\begin{abstract}
Purpose: The purpose of this study was to investigate the effect of the cognitive-linguistic intervention in people aged 80 and older. Methods: Twenty-four individuals were divided into two groups: intervention ( $83.08 \pm 2.61 \mathrm{yrs})$ and control (82.67 $\pm 2.10 \mathrm{yrs})$. The interventions involved a total of ten twice-weekly sessions. Treatment protocol consisted of various cognitive-linguistic tasks including attention, visuoperception, memory, problem-solving, executive function, and language. Results: It was found that the intervention group improved significantly in 5 domains including memory and language. Furthermore, pre and post differences in the cognitive-linguistic abilities like problem solving and language were significant between two groups. Conclusion: These findings systematically demonstrate the effect of cognitive-linguistic intervention for the elderly. The results also facilitate evidence-based and preventive cognitive-linguistic interventions, and eventually contribute to improving the quality of life in older adults.
\end{abstract}

Key Words: Aging, Cognitive-linguistic decline, Cognitive-linguistic intervention.

Received: March 24, 2018 / Revised: April 4, 2018 / Accepted: April 5, 2018

Correspondence: Mi Sook Lee, Department of Audiology \& Speech-Language Pathology, Hallym University of Graduate Studies, 427 Yeoksam-ro, Kangnam-ku, Seoul 06197, Korea

Tel: +82-70-8680-6961 / Fax: +82-2-3453-6618 / E-mail: mslee2018@hallym.ac.kr

\section{INTRODUCTION}

노화는 인간의 인지-언어 능력에 변화를 초래한다. Schaie (2005)는 종단 연구를 통해 처리속도, 추론력, 집행기능 등의 인지능력이 노화에 따라 저하된다고 보고했다. 특히, 기억력, 인 지 처리속도, 추론력은 교육수준이나 건강 상태와 상관없이 20 대 이후부터 점진적으로 저하된다(Salthouse, 2009). 이에 따라 노인의 인지-언어적 변화는 고령화 시대의 관심사로서 급부상하 고 있다. 실제로 경미한 기억력 장애가 1년 이내에 치매로 진전되 는 경우는 1 2\%에 불과하지만, 3 4년에 걸쳐 9 50\%까지 증 가된다고 보고된 바 있다(Goldman \& Morris, 2001; Miller et al., 2013).

노인의 인지-언어적 변화에 대한 논의가 확대되면서 예방적 중재의 필요성에 대한 인식도 증대되는 추세이다(Brodziak et al., 2015; Lee, 2016a). 특히, 인지-언어적 기능은 노년기 삶의
질과 직결된다는 점에서 관심이 더욱 고조되고 있다(Forte et al., 2015; Lee, 2016b).

노인을 대상으로 한 인지-언어적 중재의 필요성은 뇌 가소성 (brain plasticity)에 근거하여 설명될 수 있다. 경험에 의해 형성 되는 잠재력을 의미하는 뇌 가소성은 성인기 이후 점차 저하되 지만 평생에 걸쳐 발현되는 기능이다(Stine-Morrow et al., 2014). 이는 적응력을 유연하게 조절하는 데 기여하며, 경험한 환 경의 요구와 현재 보존된 능력 사이의 불균형에 따라 다르게 기 능한다(Payne et al., 2011). 예컨대, 환경의 요구가 현재 보존된 능력의 범위를 초과하면 적응력을 향상시키는 데 필요한 환경 적 요구에 지속적으로 부합할 수 없다(Payne et al., 2011). 새로 운 상황에 적응하거나 학습하는 능력은 적응 기능을 조절하는 능력의 집합체, 즉 인지 및 언어 능력에 의해 좌우된다(StineMorrow et al., 2014). 따라서, 뇌 가소성이 증진될수록 인지-언 어적 기능이 향상된다(Buschkuehl \& Jaeggi, 2010).

노화에 따른 인지-언어 능력의 저하를 완화하는 요인으로 
흔히 인지 보존 능력(cognitive reserve, CR)을 꼽는다(Lee, 2015c; Stern, 2009). CR이란 보완적인 뇌 연결망을 통해 인지적 수행력을 최적화 또는 극대화시키는 능력으로, 치매의 발생뿐 아니라 일반 노인의 인지-언어 능력에도 영향을 미친다(Fritsch et al., 2007; Stern, 2002). CR이 높은 노인은 병리적 양상 없이 비교적 오래 인지-언어적 기능을 보유할 수 있다(Fritsch et al., 2007). CR과 인지-언어 능력 간의 상관성을 살펴본 연구에 따르 면, 교육수준, 직업, 인지자극 활동, 다중언어의 사용 등이 $\mathrm{CR}$ 의 주요 유형에 해당하였다(Lee, 2015c). 또한 주의력, 시지각력, 기 억력, 추론력, 언어능력, 문제해결력은 $\mathrm{CR}$ 과 높은 상관성을 보 였으며, 조직화 능력, 집행기능과 $\mathrm{CR}$ 의 상관관계는 유의미하지 않았다. 정신 활동의 빈도는 인지-언어적 변화에 크게 관여하 며, 치매의 발생률을 감소시키는 데 기여한다(Verghese et al., 2003). 따라서 다양한 정신 활동을 포괄하는 인지-언어적 중재 는 노화에 따른 기능의 저하를 완화하는 데 효과적이다.

노인에 대한 인지-언어적 중재 방식은 크게 두 가지 모델로 분류된다. 첫째, 훈련(training) 모델은 특정 인지-언어 영역에 초점을 두는 중재 방식이다. 훈련을 통해 인지 및 언어의 특정 능력을 중재한 후 훈련 내용과 유사하거나 다르게 나타나는 결 과들을 평가한다(Ball et al., 2002). 둘째, 참여(engagement) 모 델은 다양하고 자극적인 환경에 개별적으로 참여함으로써 맥락 에 기반한 중재에 중점을 둔다. 복합적인 활동, 여가 활동, 사회 적 교류 등에 참여함으로써 상호관계적인 요소에 초점을 맞춘 다(Rohwedder \& Willis, 2010; Verghese et al., 2003).

노화의 부정적인 영향을 최소화하기 위한 예방적 조치로서 인지-언어적 중재의 효과성을 입증하려는 시도들이 증가하고 있다. Willis \& Caskie(2013)는 2,832명의 노인들을 대상으로 추론력, 인지 처리속도, 기억력에 대한 중재를 실시한 후 효과 성을 입증한 바 있다. 일상생활에서의 기능적인 중재에 중점 을 둔 경우도 많은데, 독서, 자서전 쓰기, 게임 활동, 여행 등이 이에 해당한다(Brodziak et al., 2015; Rebok et al., 2014). 이 밖에 컴퓨터 뇌 운동 프로그램, 사회성 자극 집단 중재, 사회적 상호작용 중심의 구어 활동 등이 시도된 바 있다(Miller et al., 2013; Pitkala et al., 2011; Shune \& Duff, 2014). 이 같은 인지언어적 중재는 노인의 삶의 질에 긍정적으로 작용한다는 보고 가 많다(Forte et al., 2015; Lee, 2016b).

인지-언어적 중재의 효과를 하위 영역별로 살펴본 연구들도 있다. 기억력과 기능적 인지능력, 문제해결력과 같은 영역의 효 과크기가 크다고 알려져 있다(Lee, 2015c). 기억력 기법 훈련, 형상화 등 기억력 중심의 중재에서는 학습능력, 즉각 및 지연 기억력의 효과가 유의미하게 나타났고(Martin et al., 2011; Zehnder et al., 2009), 뇌 가소성 기반(brain-plasticity-based) 중재에서도 기억력이 전반적으로 증진되었다(Mahncke et al., 2006b). 뇌 가소성 기반 중재는 청각적 이해력을 향상시키는 데 중점을 두고 하향 조정된 뇌의 신경조절(neuromodulatory) 구 조를 자극하는 것으로, 자극 인식, 변별, 순서화, 기억력 등의 과제를 수행하기 위한 감각적-인지적 요구에 따라 난이도가 점 점 높아지도록 구성된다(Mahncke et al., 2006b). 이를 통한 중 재 효과는 노인뿐 아니라 청장년층과 아동에까지 확대되어 검 증된 바 있다.

문제해결력은 일상의 기능적인 측면을 직접적으로 반영하기 때문에 복합적 또는 다영역적(multimodal) 활동이나 뇌 가소 성에 기반한 중재를 통해 시도하는 경우가 많다(Cavallini et al., 2015; Feng et al., 2014; Noice \& Noice, 2013; Smith et al., 2009). 실생활 중심 치료, 개별적인 가정방문 프로그램, 문 제해결 중심 신체 활동 등이 문제해결력의 향상에 효과적이라 는 보고도 있다(Ikeno, 2009; Spector et al., 2008; Torbeyns et al., 2014).

읽기를 포함한 언어 중재는 전반적인 의사소통뿐 아니라 다 양한 인지 능력, 중재에 대한 동기의식, 사회적 참여를 촉진시 키며(Wallin et al., 2018), 소리 내어 읽기, 간단한 계산, 메모나 일기 쓰기 등 일상의 쉬운 과제들을 활용하면 중재 효과가 더 커진다(Nouchi et al., 2012; Ukawa et al., 2012). 언어 중재는 상호 연계된 인지능력에도 긍정적인 영향을 미친다. 언어 과제 를 기반으로 한 중재를 실시하면 언어능력뿐 아니라 주의력, 기 억력, 집행기능 등이 향상되며(Suzuki et al., 2014), 읽기와 수 개념 중심의 중재는 추론력, 인지 처리속도 등을 증진시킨다 (Uchida \& Kawashima, 2008).

특정 프로그램을 활용한 인지-언어적 중재의 효과성을 검토 한 연구들도 있다. Kwok et al.(2013)은 '활동적 사고(active mind)'라는 인지 훈련 프로그램을 노인에게 시행한 후 주의력, 시지각력, 기억력, 추론력에 대한 효과성을 규명하였다. 구어-시 각 전환 훈련은 작업기억을 향상시키며(Osaka et al., 2012), 컴 퓨터 기반의 기억력 프로그램이 기억력, 주의력, 전반적 인지능 력에 긍정적인 영향을 미친다는 보고도 있다(Hickman et al., 2007; Mahncke et al., 2006a). 이 밖에 간섭 과제 처리 훈련은 분리주의력을 향상시키는 데 기여한다(Osaka et al., 2012).

노인에 대한 인지-언어적 중재는 고령화 추세가 급격히 진행 되고 있는 국내에서도 활발히 논의되고 있다. 그러나 중재의 필 요성에 대한 인식에도 불구하고 국내 임상 환경의 특수성 및 한계, 해당 노인 및 가족의 인식 부족 등으로 인해 적극적인 중 재나 연구가 이루어지지 않는 실정이다. 국내에서는 주로 경도 인지장애(mild cognitive impairment, $\mathrm{MCI}$ ), 치매, 뇌졸중 등 신경병리학적 질환으로 진단받은 노인에 한해 중재가 시행되고 있다. 즉, 임상 전(preclinical) 단계의 일반 노인들을 대상으로 예방적 조치로서의 인지-언어적 중재를 실시하는 사례는 매우 
드물다. 언어재활의 필요성에 대한 인식을 조사한 Park et al.(2012)의 연구에 따르면, 약 $37 \%$ 의 노인만이 중재에 긍정적 인 참여 의사를 보였다. 중재에 대한 효과성 역시 매우 제한적 으로 검토되고 있는 실정이다. 즉, 노년층을 대상으로 한 인지언어적 중재는 주로 신경병리학적 진단을 받은 노인을 대상으 로 하거나 환자군과 정상군 간의 비교를 목적으로 한 연구가 대다수이다(Cheon \& Kim, 2016; Mo et al., 2015). 최근에는 모바일 기기를 활용한 중재 프로그램을 개발하거나 인지-언어 적 중재의 효과성을 검증하기 위한 시도들이 늘어나고 있다 (Lee, 2016a; Lee et al., 2017).

요컨대 노인의 인지-언어적 중재에 대한 관심과 인식은 전 세계적으로 증폭되는 추세이나, 국내의 경우 실제적인 중재의 시행이나 효과성의 검증이 매우 미비한 실정이다. 이에 본 연구 에서는 신경학적 질환이 없는 정상 노인을 대상으로 인지-언어 적 중재를 실시하고, 중재의 효과를 각 하위 영역별로 검토해 보고자 한다. 구체적인 연구 문제는 다음과 같다.

첫째, 노인의 인지 및 언어 능력이 중재 전후에 유의한 차이 를 보이는지 살펴보고자 한다.

둘째, 인지-언어적 중재를 받은 집단과 중재를 받지 않은 집 단 간에 인지 및 언어 능력의 차이가 있는지 비교하고자 한다.

\section{MATERIALS AND METHODS}

\section{연구 대상}

본 연구는 2017년 2월부터 5월까지 서울 및 경기 지역에 거 주하는 80 세 이상의 고령 노인 총 24 명을 대상으로 하였다. 이 들은 대부분 서울시 소재 노인 복지관 1곳으로부터 표집되었 고, 서울 및 경기 지역의 자원자들도 포함되었다. 대상자들은 모두 사전에 연구의 목적 및 절차에 대한 상세한 설명을 들은 후 참여에 동의하였다. 연령의 분류 기준은 매우 다양하나, 본 연구에서는 80세 이상의 노인을 고령(old-old)으로 규정한 Grady et al.(2015)의 연구에 근거하였다.

노인 집단의 선정 기준에는 1) 만 80세 이상의 노인, 2) Korean Mini-Mental State Examination (K-MMSE)의 정상군 규준(Kang, 2006)에 의거해 정상 범주에 속하는 노인, 3) 신경 학적 질환의 병력이 없는 노인, 4) 최소 6년 이상의 교육을 받은 노인이 포함되었다. 24 명의 노인들은 인지-언어적 중재의 시행 유무에 따라 실험군 12 명과 통제군 12 명의 두 집단으로 각각 분 류되었다. 두 집단 간에 연령 $(t=0.431, p=0.671)$, 교육 연수 $(t=$ $0.208, p=0.837)$, K-MMSE 점수 $(t=-0.186, p=0.854)$ 의 차이 는 유의하지 않았다.

연령, 교육 연수, K-MMSE의 평균 및 표준편차, 성별 등 두 집단의 인구통계학적 특성은 Table 1에 제시하였다.

\section{연구 도구}

사전-사후 평가

두 집단의 인지-언어 능력을 평가하기 위한 도구로서 Cognitive-pragmatic language ability Assessment Protocol for Traumatic Brain Injury (CAPTBI) (Lee, 2013)를 적용하였다. $\mathrm{CAPTBI}$ 는 정상 노인 및 신경학적 뇌 손상 환자의 인지-언어 능력을 평가하는 도구로, 9 개 영역의 57개 문항으로 구성되어 있다. 본 연구에서는 인지 관련 하위 검사 중 주의력, 시지각력, 기억력, 문제해결력, 집행기능 등 총 5 개 능력을 평가하였다. 언 어 영역 중에서는 비유언어, 기능/상징언어 능력을 평가하였다. 일대일 직접 평가를 통해 검사자가 지시문 및 자극을 시·청각적 으로 제시한 후 피검자의 반응을 기록하였다. 3점 척도 $(0,1,2$ 점) 를 적용하여 채점하되, 문항에 따라 정반응 개수, 정·오반응 여 부, 명시화된 기준 등에 근거하여 점수를 산정하였다. 영역별 점 수 범위는 주의력 및 기억력 0 8점, 시지각력 0 6점, 문제해결 력 0 14점, 집행기능 0 4점, 비유언어 0 28점, 기능/상징언어 0 8점으로, 점수가 높을수록 해당 인지-언어 능력이 높은 것 으로 간주한다.

사전-사후 평가에 활용된 인지-언어 능력의 하위 영역 및 총 점은 Table 2 와 같다.

\section{중 재}

실험군의 중재를 위해 '실어증 및 인지-의사소통 환자를 위 한 언어치료 워크북(Lee, 2015b)'을 활용하였다. 인지-언어적 중재는 총 6 개 하위 영역으로 분류되었는데, 복합적인 능력을 요하는 영역에서는 해당 과제를 중복적으로 포함하였다. 중재 에 활용된 모든 문항은 사전-사후 평가 문항과 전혀 중복되지 않았다.

인지-언어적 중재에 활용된 과제는 다음과 같다. 주의력은 시각적 주의력 및 청각적 주의력 과제, 시지각력은 순서대로 선 긋기, 삼각형 만들기, 시계 그리기 과제, 기억력은 시각적 단기기 억과 청각적 단기기억 과제를 시행하였다. 문제해결력은 지문 읽고 나열하기, 해결방법 유추하기 과제를 사용하였다. 집행기 능이 문제해결, 개념의 형성 및 추론 등의 복합적인 작용을 통

Table 1. Demographic characteristics in two groups

\begin{tabular}{lcc}
\hline \multicolumn{1}{c}{ Characteristic } & $\begin{array}{c}\text { Intervention group } \\
(\mathrm{n}=12)\end{array}$ & $\begin{array}{c}\text { Control group } \\
(\mathrm{n}=12)\end{array}$ \\
\hline Age $(\mathrm{yr})$ & $83.08( \pm 2.61)$ & $82.67( \pm 2.10)$ \\
Gender $(\mathrm{M}: \mathrm{F})$ & $5: 7$ & $4: 8$ \\
Education level $(\mathrm{yr})$ & $8.25( \pm 3.05)$ & $8.00( \pm 2.83)$ \\
K-MMSE & $21.92( \pm 2.19)$ & $22.08( \pm 2.19)$ \\
\hline
\end{tabular}

K-MMSE: Korean Mini-Mental State Examination 
해 기능한다는 점을 고려하여 차이점 유추하기, 공통점 유추하 기, 지문 읽고 나열하기, 해결방법 유추하기 과제를 통해 중재 하였다(Busch et al., 2005; Ewing-Cobbs et al., 2004; Lee, 2016b). 화용언어는 개념의 통합과 추상적 사고, 고차원적 언어 의 해석 및 표현과 관련되므로 차이점 유추하기, 공통점 유추 하기, 속담 완성하기, 비유언어 이해하기 과제를 활용하였다 (Channon \& Watts, 2003; Douglas et al., 2007; Lee, 2016b). 인지-언어적 중재를 위한 과제는 Table 2에 제시하였다.

\section{연구 절차}

\section{실험군}

실험군을 대상으로 사전 평가, 중재, 사후 평가를 시행하였고, 5 7주에 걸쳐 총 3단계로 진행하였다. 중재를 시작하기 전 1회 의 사전 평가를 실시한 후 주 2 회기씩 5 주간 총 10 회기의 중재 를 실시하였다. 대상자가 80세 이상의 고령 노인이므로 피로도 및 주의력 등을 감안하여 한 회기당 30 분씩 할당하였다. 10 회기 의 중재를 완료한 후 일주일 이내에 사전 평가와 동일한 방식으
로 1회의 사후 평가를 시행하였다. 사전-사후 평가는 평균 30 40분 정도 소요되었다. 평가 및 중재는 조용한 방에서 연구자가 일대일로 진행하였다.

중재 시에는 매 회기마다 집행기능을 제외한 5 개 영역의 과 제들을 고루 시행하되, 한 영역당 1 2개의 과제로 제한하였다. 집행기능에 해당하는 과제들은 문제해결력 및 언어 영역에 속 한 일부 과제들과 중복되므로 별도로 시행하지 않았다. 모든 과제는 난이도에 따라 수준 1 과 수준 2 로 구분되었다. 예를 들 어, 집행기능 및 화용언어의 '차이점 유추하기' 과제에서 수준 1 은 두 낱말(예: 의자-침대)에 대한 차이를, 수준 2 는 세 낱말 (예: 컵-그릇-냄비) 간의 차이를 유추하도록 구성되었다. 또한, 문제해결력 및 집행기능의 ‘해결방법 유추하기' 과제는 상황적 복잡성에 따라 수준 1(예: 심이 부러진 연필)과 수준 2(예: "빈 지갑'과 '자판기 사용'을 연결하는 상황)로 이원화하였다. 각 영 역별로 수준 1의 과제들을 반복적으로 시행하여 $80 \%$ 이상의 정반응을 보이는 시점에서 수준 2 의 과제들을 진행하였다. 본 연구에 사용된 중재 과제의 세부 내용은 Table 3에 제시하였다.

중재 시 오반응을 보일 경우 제시되는 단서의 유형은 다음과

Table 2. Cognitive-linguistic domains of evaluation and intervention tasks

\begin{tabular}{lrll}
\hline \multicolumn{1}{c}{ Evaluation } & \multicolumn{2}{c}{ Intervention task } \\
\cline { 1 - 2 } Domain & Score & \\
Attention & 8 & Visual attention, auditory attention \\
Visuoperception & 6 & Drawing line in regular sequence, forming triangle, drawing clock, finding shortcut, drawing face \\
Memory & 8 & Visual STM, auditory STM \\
Problem solving & 14 & Explaining solution, inferring solution \\
Executive function & 4 & Inferring difference, inferring similarity, explaining solution, inferring solution \\
Pragmatic language & 36 & Inferring difference, inferring similarity, completing proverb, comprehending figurative language \\
\hline
\end{tabular}

STM: short-term memory

Table 3. Details of intervention tasks

\begin{tabular}{ll}
\hline \multicolumn{1}{c}{ Intervention tasks } & \multicolumn{1}{c}{ Details } \\
\hline Visual attention & Marking on pictures of shape/color/fruit/item/animal, finding differences in 2-3 pictures \\
Auditory attention & Responding to 'ga' sound in sentence, answering questions, calculating \\
Drawing a line in regular sequence & Drawing a line in regular sequence of number/consonant/number \& consonant \\
Forming triangles & Forming small triangles in a large triangle/square \\
Drawing a clock & Marking time on a clock (with or without numbers) \\
Finding a shortcut & Finding and marking a shortcut in a map \\
Drawing a face & Drawing a person's face \\
Visual STM & Recalling picture/word presented visually \\
Auditory STM & Recalling number/word presented auditorily \\
Explaining solution & Explaining solution presented in paragraph \\
Inferring solution & Inferring solution of photo/paragraph \\
Inferring difference & Inferring differences in 2-3 words \\
Inferring similarity & Inferring similarities in 2-3 words \\
Completing proverb & Completing and saying proverb (multiple-choice/subjective) \\
Comprehending figurative language & Comprehending meaning of simile/metaphor \\
\hline
\end{tabular}

STM: short-term memory 
같다. 첫째, 시·청각적 자극을 반복적으로 제시한다. 시각적 및 청각적 단기기억, 청각적 주의력 과제가 이에 해당한다. 둘째, 자극의 일부를 단계적으로 보완하여 제시한다. 이는 시계 그리 기, 지름길 찾기, 얼굴 그리기 과제에 적용된다. 예컨대 얼굴 그 리기 과제에서 오반응을 보일 경우 얼굴의 일부를 그려 다시 제시한 후 나머지를 완성하도록 요구한다. 셋째, 정반응을 유추 할 수 있도록 상황에 대해 부연 설명을 하거나 관련된 단어를 제시한다. 지문 읽고 나열하기, 해결방법 유추하기, 차이점 및 공통점 유추하기, 속담 완성하기, 비유언어 이해하기 과제가 이 에 해당한다. 넷째, 자극이나 보기의 수를 단계적으로 축소하 여 제시한다. 여기에는 순서대로 선 긋기, 시각적 및 청각적 단기 기억, 속담 완성하기, 비유언어 이해하기 과제가 해당된다. 다섯 째, 보기가 없는 과제에 오반응을 보일 경우 보기를 제시한다. 이는 삼각형 만들기, 시계 그리기, 지름길 찾기, 지문 읽고 나열 하기, 해결방법 유추하기, 차이점 및 공통점 유추하기 과제에서 적용된다.

\section{통제군}

통제군을 대상으로 사전-사후 평가를 각각 1회씩 시행하였으 며, 실험군과의 비교를 위해 사전 평가 후 5 7주 이내에 사후 평가를 실시하였다. 평가는 평균 30 40분 정도 소요되었다. 사 전-사후 평가 사이에 통제군을 대상으로 한 인지-언어적 중재 는 전혀 시행하지 않았다.

\section{통계 분석}

본 연구에는 사전-사후 비교 설계(pretest-posttest design)가 활용되었고, 자료 분석을 위해 대상군에게 적용한 사전-사후 평가의 각 영역별 점수를 산출하였다. 통계분석 프로그램으로 는 SPSS 21.0 version (Statistical Product and Service Solution 21.0) (IBM Corp., Armonk, NY, USA)을 활용하였다. 연 령과 교육 연수, $\mathrm{K}-\mathrm{MMSE}$ 점수상 집단 간의 차이가 있는지 확 인하기 위해 독립표본 $t$-검정(independent samples $t$-test)을 사 용하였고, 기술통계 결과를 통해 하위 영역별 수행력을 살펴보 았다. 실험군을 대상으로 실시한 중재 전후의 수행력 차이를 검
증하기 위해 비모수적 통계방법인 윌콕슨 결합-조 기호-순위 검정(Wilcoxon matched-pairs signed-ranks test)을 활용하였 다. 사전-사후 평가에서 두 집단 간 수행력의 차이는 맨 휘트니 유 검정(Mann-Whitney U test)을 통해 비교하였다.

\section{RESULTS}

\section{중재 전후 인지-언어 능력의 비교}

실험군의 인지-언어 능력이 중재 전후에 유의한 차이를 보이 는지 비교하였다(Table 4). 그 결과, 시지각력( $\mathrm{Z}=-2.976, p<$ $0.01)$, 기억력 $(\mathrm{Z}=-2.611, p<0.01)$, 문제해결력 $(\mathrm{Z}=-2.744, p<$ $0.01)$, 집행기능 $(\mathrm{Z}=-2.460, p<0.05)$, 언어능력 $(\mathrm{Z}=-3.090, p<$ 0.01) 등 5개 하위 영역에서 유의한 차이가 있었다. 즉, 노인의 시 지각력, 기억력, 문제해결력, 집행기능, 언어능력은 인지-언어적 중재를 통해 수행력이 향상되었다. 반면에, 주의력은 중재 후에 수행력이 다소 향상되었으나 유의미한 차이는 나타나지 않았다 $(\mathrm{Z}=-1.732, p=0.083)$

\section{집단 간 사전-사후 차이 비교}

실험군과 통제군의 사전-사후 차이를 비교한 결과는 Table 5 와 같다. 주의력 $(\mathrm{U}=49.500, p<0.05)$, 시지각력 $(\mathrm{U}=12.000$, $p<0.01)$, 기억력 $(\mathrm{U}=16.500, p<0.01)$, 문제해결력 $(\mathrm{U}=$ $26.000, p<0.01)$, 언어능력(U = 4.000, $p<0.01)$ 등 5개 영역 은 두 집단 간의 사전-사후 차이가 유의미한 것으로 분석되었 다. 즉, 실험군의 사전-사후 차이가 통제군의 차이보다 유의하 게 큰 것으로 나타났다. 그러나, 두 집단 간에 집행기능의 사 전-사후 차이는 유의하지 않았다( $\mathrm{U}=47.500, p=0.119)$.

\section{DISCUSSIONS}

본 연구에서는 정상 노인을 대상으로 인지-언어적 중재를 실 시한 후 각 하위 영역별로 중재 효과를 살펴보았다. 이를 위해 노인의 인지 및 언어 능력이 중재 전후에 유의한 차이를 보이는 지 알아보았다. 또한, 중재 여부에 따라 노인의 인지 및 언어 능

Table 4. Cognitive-linguistic abilities in pre and post intervention $(n=12)$

\begin{tabular}{lrrrr}
\hline \multicolumn{1}{c}{ Domain } & Pre scores & Post scores & $Z$ & \multicolumn{1}{c}{$p$} \\
\hline Attention & $5.08(1.00)$ & $5.33(0.98)$ & -1.732 & 0.083 \\
Visuoperception & $2.08(0.51)$ & $3.92(1.16)$ & -2.976 & $0.003^{\dagger}$ \\
Memory & $5.08(1.00)$ & $7.00(1.28)$ & -2.611 & $0.009^{\dagger}$ \\
Problem solving & $10.42(0.51)$ & $12.92(1.62)$ & -2.744 & $0.006^{\dagger}$ \\
Executive function & $3.08(0.79)$ & $3.83(0.39)$ & -2.460 & $0.014^{*}$ \\
Language & $26.08(4.29)$ & $31.33(2.74)$ & -3.090 & $0.002^{\dagger}$ \\
\hline
\end{tabular}

Pre \& post scores: mean ( \pm standard deviation). $Z$ value: a continuity correction to account for the fact that we use a continuous probability function (the normal distribution). ${ }^{*} p<0.05,{ }^{\dagger} p<0.01$ 
Table 5. Comparison of cognitive-linguistic differences between two groups

\begin{tabular}{lcccc}
\hline \multicolumn{1}{c}{ Domain } & Intervention group $(\mathrm{n}=12)$ & Control group $(\mathrm{n}=12)$ & $\mathrm{U}$ & $\mathrm{p}$ \\
\hline Attention & $0.25(0.43)$ & $-0.08(0.28)$ & 49.500 & $0.045^{*}$ \\
Visuoperception & $1.83(0.90)$ & $0.25(0.43)$ & 12.000 & $0.000^{\dagger}$ \\
Memory & $1.92(1.61)$ & $-0.25(0.60)$ & 16.500 & $0.001^{\dagger}$ \\
Problem solving & $2.50(1.76)$ & $0.17(0.37)$ & 26.000 & $0.004^{\dagger}$ \\
Executive function & $0.75(0.72)$ & $0.25(0.60)$ & 47.500 & 0.119 \\
Language & $5.25(1.74)$ & $0.08(1.50)$ & 4.000 & $0.000^{\dagger}$ \\
\hline
\end{tabular}

Score: mean ( \pm standard deviation) of pre-post differences in each group, $U$ value: a value compared against the normal distribution to calculate the confidence level. ${ }^{*} p<0.05,{ }^{\dagger} p<0.01$

력에 차이가 있는지 분석하였다.

본 연구에 활용된 중재 방법은 훈련 모델(Ball et al., 2002)에 근거하였다. Nouchi et al.(2012)에 따르면, 인지-언어 훈련이 전 두 및 전전두(prefrontal) 피질, 배측면(dorsolateral) 피질, 두정 연합 피질에 대한 자극을 강화해 노인의 기억력, 집행기능, 인지 처리속도 등의 저하를 완화시킨다. 본 연구에서는 주로 기능적 인 상황에 대한 문제해결이나 유추, 일상생활에서 흔히 접할 수 있는 시-청각 자극에 중점을 둔 과제를 활용하였는데, 이는 과제의 친숙도가 수행력의 큰 변수로 작용하는 노년층의 특징 을 반영한 결과이다(Lee, 2016a; Lee \& Kim, 2012; Nouchi et al., 2012). 특히 사진, 그림 등의 시각적 자극과 그리기 등의 과 제가 전대상피질(anterior cingulate cortex), 좌우측 하두정소 엽(inferior parietal lobule), 우측 상두정소엽(superior parietal lobule)을 활성화시킨다는 점도 고려하였다(Osaka et al., 2012).

주의력과 기억력은 노화의 영향을 받는 기초적인 인지기능이 다. 언어능력이나 문제해결력과 같은 고차원적 기능 역시 나이 가 들수록 저하되는데, 이로 인해 의사소통이나 문제해결 과제 를 처리할 때 주의력, 기억력 등의 기초적인 인지기능에 더 의 존하게 된다(Ibrahimagic et al., 2017). 따라서 기초적인 인지기 능이 저하될수록 필연적으로 고차원적 능력도 떨어진다. 이는 인지-언어 능력이 개별적으로 기능하지 않고 복합적으로 연계되 어 있음을 뒷받침하는 근거가 된다(Ibrahimagic et al., 2017; Lee, 2016a). 예컨대 노인을 대상으로 다영역적 인지-언어 중재 를 실시하면 문제해결력과 언어능력이 동시에 향상된다(Noice \& Noice, 2009; Noice \& Noice, 2013). 본 연구에서 실험군을 대상으로 인지-언어적 중재를 실시할 때 이 같은 상호 연계성 을 최대한 활용하였다.

중재 이후 실험군은 시지각력, 기억력, 문제해결력, 집행기능, 언어능력 등 5 개 하위 영역에서 유의한 향상을 보였다. 또한 주 의력, 시지각력, 기억력, 문제해결력, 언어능력 등 5 개 영역에서 실험군의 사전-사후 차이가 통제군에 비해 유의하게 큰 것으 로 나타났다.

노인들에게 인지-언어적 중재를 시행하면 시지각력, 기억력 과 같은 기초적인 인지능력이 향상된다고 알려져 있다(Kwok et al., 2013). 예를 들어, 컴퓨터를 활용한 각종 인지 게임은 노 인의 시공간력을 향상시키는 데 매우 효과적이다(Peretz et al., 2011). 노인을 대상으로 한 기억력 중재의 효과성을 규명한 연 구는 상대적으로 많은 편이다(Cavallini et al., 2015; Noice \& Noice, 2009; Smith et al., 2009). 이는 MCI, 치매 등 신경학적 질환의 초기부터 기억력이 저하된다는 점에 기인한다. 2000년 부터 2015년까지의 연구들을 대상으로 인지-언어적 중재의 효 과성을 살펴본 메타분석에서는 기억력의 효과크기가 공통적으 로 크게 나타났다(Lee, 2016a). 구어-시각 전환 훈련과 인지자 극 훈련은 작업기억을 향상시키는 데 유용하다(Osaka et al., 2012; Wilson et al., 2002). 기억력 기법 훈련이나 형상화 등을 활 용한 기억력 중재가 노인의 학습능력, 즉각 및 지연 기억력을 향 상시켰다는 보고도 있다(Martin et al., 2011; Zehnder et al., 2009). 기억력의 중재는 현재의 능력을 보존할 뿐 아니라 인지-언 어적 수행력을 향상시키는 데에도 잠재적으로 기여하기 때문에, 예방적 조치로서 반드시 고려되어야 할 영역 중 하나이다(Gross et al., 2012).

문제해결력은 일상생활에서 직면하는 대부분의 상황에 요구 되는 기능이므로 인지-언어적 중재 시 주요 목표로 삼는 경우 가 많다. 또한 인지 및 언어와 관련된 과제들의 대부분은 문제 해결적인 기능을 반영하고 있어 중재 시 더욱 복합적이고 다양 식적인 특성을 띤다(Cavallini et al., 2015; Feng et al., 2014). 특히, 노인을 대상으로 한 인지-언어적 중재 시에는 실생활 중 심의 기능적인 치료나 생활양식 관련(lifestyle-related) 예방 및 중재가 유용하다(Norton et al., 2014; Torbeyns et al., 2014; Wallin et al., 2018). 본 연구에서도 일상의 다양한 사물이나 상 황(예: 끈이 풀린 운동화, 진료 예약)에 대한 해결방법을 유추하 는 데 중점을 두었다.

집행기능은 일상생활 전반과 직결되는 복합적인 인지능력으 로, 노인을 대상으로 한 예방적 중재에서 특히 강조되는 영역이 다(Dawson et al., 2014; Suzuki et al., 2014). 다양한 두뇌 훈련 게임은 노년층의 전반적인 인지-언어 능력을 보존하는 데 기여 하는데, 집행기능과 인지 처리속도를 향상시키는 데 더욱 효과 적이다(Nouchi et al., 2012). 또한 기능적이고 복합적인 인지- 
언어 과제로 구성된 중재는 노인의 집행기능을 향상시킨다 (Dawson et al., 2014). 본 연구에서 집행기능은 해결방법 유추 하기 및 설명하기, 차이점 및 공통점 유추하기 등 문제해결력과 화용언어에 대한 중재를 통해 효과성을 검토하였다. 두 영역에 서 요구되는 복합적인 처리능력이 필연적으로 집행기능의 중재 효과를 높이는 데 기여한 것으로 보인다.

언어능력에 대한 중재는 인지 영역과 연계시키거나 기능적인 의사소통 능력을 증진하는 데 초점을 두는 경우가 많다. 직접 적인 중재가 아니더라도 언어능력과 상관성이 높은 인지 영역 을 중재한 후 언어능력에 대한 효과를 분석하기도 한다(Edwards et al., 2015). 본 연구에서도 해결방법 유추하기, 차이점 및 공통점 유추하기, 속담 완성하기, 비유언어 이해하기 등 추 론력, 문제해결력과 같은 복합적인 인지능력과 연계된 언어 과 제를 활용하였다. 이와 유사한 맥락으로 언어 과제에 중점을 둔 중재가 노인의 언어능력뿐 아니라 주의력, 기억력, 집행기능 등 전반적인 인지기능을 향상시킨다는 보고도 있다(Suzuki et al., 2014). 예컨대 계산하기와 소리 내어 읽기 과제는 집행기능, 인지 처리속도에 긍정적인 영향을 미친다(Uchida \& Kawashima, 2008). 이는 노인뿐 아니라 치매 등 신경병리학적 환자들의 경우 에도 적용된다고 알려져 있다(Kulason et al., 2016).

한편, 본 연구에서 주의력의 중재 효과는 미미한 것으로 나 타났으며, 실험군과 통제군 간에 집행기능의 사전-사후 차이가 유의미하지 않았다. 이는 여러 중재 프로그램들을 분석한 연구 에서 주의력과 집행기능의 효과크기가 유의미하지 않게 나타 난 결과와 일치한다(Lee, 2016a). 본 연구의 주의력 과제는 삼 각형, 사각형, 원 등의 도형과 빨강, 파랑, 초록, 노랑 등의 색깔 이 다양하게 활용되어 시각적으로 요구되는 인지적 부담이 적 지 않다. 연령이 높을수록 시각적 요구나 자극을 처리하는 데 있어 효율성이 떨어진다는 점을 고려할 때, 이 같은 인지적 부담 이 본 연구의 중재 효과에 영향을 미친 것으로 보인다(Mozolic et al., 2011; Vohn, 2008). 또한 청각적 자극을 듣고 구어나 행 동으로 반응하는 과제(예: 단락을 듣고 '가' 소리가 나면 책상을 치기)의 경우 주의력을 전환하는 데 따르는 어려움이 변수로 작용했을 수 있다(Lee, 2015a; Vohn, 2008). 전전두 피질에서 주로 관장하는 집행기능은 주의력, 억제능력, 작업기억 등을 포 괄한다. 노화로 인해 전전두 피질의 크기와 두께가 축소되면 주의력과 집행기능이 동시에 저하될 수밖에 없다(Healey et al., 2008). 요컨대 주의력과 집행기능이 연계된 기능적·신경학 적 기제가 본 연구의 일관적인 중재 효과를 기대하기 어렵게 한 것으로 보인다.

본 연구 결과의 임상적 함의는 다음과 같다. 첫째, 노년층에 대한 인지-언어적 중재의 효과를 체계적으로 규명하는 데 기여 하였다. 둘째, 인지-언어 능력이 복합적으로 연계되어 있고, 다
양식적 또는 교차양식적(cross-modal) 특성을 띄는 점을 고려 함으로써 인지-언어적 중재 시 활용 가능한 임상적 지침으로서 의 역할을 하게 될 것이다. 셋째, 노화에 따른 인지-언어 능력의 변화에 대한 예방적 조치로서 적극적인 중재의 필요성을 환기시 킴으로써 고령화 사회의 삶의 질을 제고하는 데 기여할 것이다.

본 연구는 다음과 같은 면에서 몇몇 한계점을 지닌다. 첫째, 본 연구에서는 실험군을 대상으로 5 주간 총 10 회기의 중재를 실시하였다. 정상 노인을 대상으로 한 인지-언어적 중재의 효 과를 명확히 검증하기 위해서는 보다 장기간에 걸친 중재가 요 구된다. 둘째, 노인 집단의 연령 범위를 세분화한 후속 연구가 필요하다. 노년층의 인지-언어적 변화를 살펴본 종단 연구에서 '연령'은 교육 연수, 사회 활동 등과 함께 주요한 영향 요인으로 꼽혔다(Lee, 2015a). 따라서 상대적으로 젊은 노년층과 고령층, 초고령층 등 노년을 시기별로 세분화해 인지-언어적 중재의 효 과성을 검토할 필요가 있다(Chen et al., 2017). 셋째, 본 연구에 서 활용한 중재 과제는 총 2개의 수준으로 구성되어 있으며, 지 면이나 구어 지시를 통해 제시되는 시각적 및 청각적 자극에 반응하는 방식이다. 후속 연구에서는 노화에 민감한 과제를 수 준별로 세분화하고, 자극을 제시하는 매체나 양식을 보다 다양 화해야 할 것이다.

중심 단어 : 고령화·인지-언어적 저하·인지-언어적 중재.

\section{REFERENCES}

Ball, K., Berch, D. B., Helmers, K. F., Jobe, J. B., Leveck, M. D., Marsiske, M., et al.; Advanced Cognitive Training for Independent and Vital Elderly Study Group. (2002). Effects of cognitive training interventions with older adults: A randomized controlled trial. Journal of the American Medical Association, 288(18), 2271-2281.

Brodziak, A., Wolińska, A., Kołat, E., \& Różyk-Myrta, A. (2015). Guidelines for prevention and treatment of cognitive impairment in the elderly. Medical Science Monitor, 21, 585-597.

Busch, R. M., Booth, J. E., McBride, A., Vanderploeg, R. D., Curtiss, G., \& Duchnick, J. J. (2005). Role of executive functioning in verbal and visual memory. Neuropsychology, 19(2), 171-180.

Buschkuehl, M. \& Jaeggi, S. M. (2010). Improving intelligence: A literature review. Swiss Medical Weekly, 140(19-20), 266-272.

Cavallini, E., Bottiroli, S., Capotosto, E., De Beni, R., Pavan, G., Vecchi, T. et al. (2015). Self-help memory training for healthy older adults in a residential care center: Specific and transfer effects on performance and beliefs. International Journal of Geriatric Psychiatry, 30(8), 870-880.

Channon, S. \& Watts, M. (2003). Pragmatic language interpretation after closed head injury: Relationship to executive functioning. Cognitive Neuropsychiatry, 8(4), 243-260.

Chen, X., Hertzog, C., \& Park, D. C. (2017). Cognitive predictors of everyday problem solving across the lifespan. Gerontology, 63(4), 372-384.

Cheon, J. M. \& Kim, W. S. (2016). The effects of group language interventions utilizing integrative art therapy on communication and cognition abilities of elderly people with mild neurocognitive disorder. Journal of Speech-Language and Hearing Disorders, 25(4), 165-185.

Dawson, D., Richardson, J., Troyer, A., Binns, M., Clark, A., Polatajko, H., et al. (2014). An occupation-based strategy training approach to managing age-related executive changes: A pilot randomized controlled 
trial. Clinical Rehabilitation, 28(2), 118-127.

Douglas, J. M., Bracy, C. A., \& Snow, P. C. (2007). Exploring the factor structure of the La Trobe Communication Questionnaire: Insights into the nature of communication deficits following traumatic brain injury. Aphasiology, 21(12), 1181-1194.

Edwards, J. D., Valdés, E. G., Peronto, C., Castora-Binkley, M., Alwerdt, J., Andel, R., et al. (2015). The efficacy of inSight cognitive training to improve useful field of view performance: A brief report. The Journals of Gerontology, Series B: Psychological Sciences and Social Sciences, 70(3), 417-422.

Ewing-Cobbs, L., Prasad, M. R., Landry, S. H., Kramer, L., \& DeLeon, R. (2004). Executive functions following traumatic brain injury in young children: A preliminary analysis. Developmental Neuropsychology, 26(1), 487-512.

Feng, W., Li, C., Chen, Y., Cheng, Y., \& Wu, W. (2014). Five-year follow-up study of multi-domain cognitive training for healthy elderly community members. Shanghai Archives of Psychiatry, 26(1), 30-41.

Forte, R., Boreham, C. A., De Vito, G., \& Pesce, C. (2015). Health and quality of life perception in older adults: The joint role of cognitive efficiency and functional mobility. International Journal of Environmental Research and Public Health, 12(9), 11328-11344.

Fritsch, T., McClendon, M. J., Smyth, K. A., Lerner, A. J., Friedland, R. P., \& Larsen, J. D. (2007). Cognitive functioning in healthy aging: The role of reserve and lifestyle factors early in life. The Gerontologist, 47(3), 307322 .

Goldman, W. P. \& Morris, J. C. (2001). Evidence that age-associated memory impairment is not a normal variant of aging. Alzheimer Disease and Associated Disorders, 15(2), 72-79.

Grady, K. L., Naftel, D. C., Myers, S., Dew, M. A., Weidner, G., Spertus, J. A., et al. (2015). Change in health-related quality of life from before to after destination therapy mechanical circulatory support is similar for older and younger patients: Analyses from INTERMACS. The Journal of Heart and Lung Transplantation, 34(2), 213-221.

Gross, A. L., Parisi, J. M., Spira, A. P., Kueider, A. M., Ko, J. Y., Saczynski, J. S., et al. (2012). Memory training interventions for older adults: A meta-analysis. Aging and Mental Health, 16(6), 722-734.

Healey, M. K., Campbell, K. L., \& Hasher, L. (2008). Cognitive aging and increased distractibility: Costs and potential benefits. Progress in Brain Research, 169, 353-363.

Hickman, J. M., Rogers, W. A., \& Fisk, A. D. (2007). Training older adults to use new technology. The Journals of Gerontology, Series B: Psychological Sciences and Social Sciences, 62(Spec 1), 77-84.

Ibrahimagic, A., Zunic, L. J., Ibrahimagic, O. C., Smajlovic, D., \& Rasidovic, M. (2017). Receptive vocabulary and cognition of elderly people in institutional care. Materia Socio-Medica, 29(2), 124-128.

Ikeno, T. (2009). A pilot study of preventive home visits applying occupational therapeutic theory for improving functional capacity (unpublished doctoral dissertation). Hokkaido University, Sapporo.

Kang, Y. W. (2006). A normative study of the Korean-Mini Mental State Examination (K-MMSE) in the elderly. Korean Journal of Psychology, 25(2), 1-12.

Kulason, K., Nouchi, R., Hoshikawa, Y., Noda, M., Okada, Y., \& Kawashima, R. (2016). The beneficial effects of cognitive training with simple calculation and reading aloud in an elderly postsurgical population: Study protocol for a randomized controlled trial. Trials, 17, 334.

Kwok, T., Wong, A., Chan, G., Shiu, Y. Y., Lam, K. C., Young, D., et al. (2013). Effectiveness of cognitive training for Chinese elderly in Hong Kong. Clinical Interventions in Aging, 8, 213-219.

Lee, H. S., Hyeon, J. I., Kim, J. W., \& Choi, Y. G. (2017). A study of the reactivity of the elderly when playing cognitive rehabilitation games on tablet devices. Journal of Speech-Language and Hearing Disorders, 26(2), 57-65.

Lee, M. S. (2013). Development and application of cognitive-pragmatic language ability assessment protocol for traumatic brain injury (CAPTBI) (doctoral dissertation). Yonsei University, Seoul.
Lee, M. S. (2015a). A longitudinal study on cognitive-pragmatic language in normal aging: Demographic and experiential influences. Journal of the Korea Gerontological Society, 35(3), 797-811.

Lee, M. S. (2015b). Linguistic workbook for aphasia and cognitive-communicative disorder (No. 10-1518680). Daejeon: Korean Intellectual Property Office.

Lee, M. S. (2015c). Meta-analysis of correlation between cognitive-linguistic ability and cognitive reserve in normal aging. Journal of Korean Contents Society, 15(11), 359-373.

Lee, M. S. (2016a). Cognitive-linguistic intervention programs on normal aging: A systematic review \& meta-analysiss. Journal of the Korea Gerontological Society, 36(1), 79-101.

Lee, M. S. (2016b). The relationship between cognitive-linguistic ability and quality of life in older adults. Journal of Special Education and Rehabilitation Science, 55(4), 143-161.

Lee, M. S. \& Kim, H. (2012). Cognitive-pragmatic language assessment for normal aging: Study of assessment tools and content validity. The Journal of the Korea Contents Association, 12(5), 280-292.

Mahncke, H. W., Bronstone, A., \& Merzenich, M. M. (2006a). Brain plasticity and functional losses in the aged: Scientific bases for a novel intervention. Progress in Brain Research, 157, 81-109.

Mahncke, H. W., Connor, B. B., Appelman, J., Ahsanuddin, O. N., Hardy, J. L., Wood, R. A., et al. (2006b). Memory enhancement in healthy older adults using a brain plasticity-based training program: A randomized, controlled study. Proceedings of the National Academy of Sciences of the United States of America, 103(33), 12523-12528.

Martin, M., Clare, L., Altgassen, A. M., Cameron, M. H., \& Zehnder, F. (2011). Cognition-based interventions for healthy older people and people with mild cognitive impairment. The Cochrane Database of Systematic Reviews, (1), CD006220.

Miller, K. J., Dye, R. V., Kim, J., Jennings, J. L., O’Toole, E., Wong, J., et al. (2013). Effect of a computerized brain exercise program on cognitive performance in older adults. The American Journal of Geriatric Psychiatry, 21(7), 655-663.

Mo, K. O., Sung, J. E., \& Jeong, J. H. (2015). The effects of semantic feature analysis treatment on naming performance in Korean individuals with early dementia of the Alzheimer's type: Using a familiarity of nouns scale. Communication Sciences and Disorders, 20(1), 34-47.

Mozolic, J. L., Long, A. B., Morgan, A. R., Rawley-Payne, M., \& Laurienti, P. J. (2011). A cognitive training intervention improves modality-specific attention in a randomized controlled trial of healthy older adults. $\mathrm{Neu}$ robiology of Aging, 32(4), 655-668.

Noice, H. \& Noice, T. (2009). An arts intervention for older adults living in subsidized retirement homes. Neuropsychology, Development and Cognition. Section B: Aging, Neuropsychology and Cognition, 16(1), 5679

Noice, H. \& Noice, T. (2013). Extending the reach of an evidence-based theatrical intervention. Experimental Aging Research, 39(4), 398-418.

Norton, S., Matthews, F. E., Barnes, D. E., Yaffe, K., \& Brayne, C. (2014). Potential for primary prevention of Alzheimer's disease: An analysis of population-based data. The Lancet. Neurology, 13(8), 788-794.

Nouchi, R., Taki, Y., Takeuchi, H., Hashizume, H., Nozawa, T., Sekiguchi, A., et al. (2012). Beneficial effects of reading aloud and solving simple arithmetic calculations (learning therapy) on a wide range of cognitive functions in the healthy elderly: Study protocol for a randomized controlled trial. Trials, 13, 32 .

Osaka, M., Otsuka, Y., \& Osaka, N. (2012). Verbal to visual code switching improves working memory in older adults: An fMRI study. Frontiers in Human Neuroscience, 6, 24.

Park, H., Shin, H. J., \& Son, M. D. (2012). A study of language disorders and language rehabilitation perceptions in elderly. Journal of Speech and Hearing Disorders, 21(4), 227-247.

Payne, B. R., Jackson, J. J., Noh, S. R., \& Stine-Morrow, E. A. (2011). In the zone: Flow state and cognition in older adults. Psychology and Aging, 26(3), 738-743. 
Peretz, C., Korczyn, A. D., Shatil, E., Aharonson, V., Birnboim, S., \& Giladi, N. (2011). Computer-based, personalized cognitive training versus classical computer games: A randomized double-blind prospective trial of cognitive stimulation. Neuroepidemiology, 36(2), 91-99.

Pitkala, K. H., Routasalo, P., Kautiainen, H., Sintonen, H., \& Tilvis, R. S. (2011). Effects of socially stimulating group intervention on lonely, older people's cognition: A randomized, controlled trial. The American Journal of Geriatric Psychiatry, 19(7), 654-663.

Rebok, G. W., Ball, K., Guey, L. T., Jones, R. N., Kim, H. Y., King, J. W., et al.; ACTIVE Study Group. (2014). Ten-year effects of the advanced cognitive training for independent and vital elderly cognitive training trial on cognition and everyday functioning in older adults. Journal of the American Geriatrics Society, 62(1), 16-24

Rohwedder, S. \& Willis, R. J. (2010). Mental retirement. Journal of Economic Perspectives, 24(1), 119-138.

Salthouse, T. A. (2009). When does age-related cognitive decline begin? Neurobiology of Aging, 30(4), 507-514.

Schaie, K. W. (2005). Developmental Influences on Adult Intelligence: The Seattle Longitudinal Study. (1st ed.). (pp. 112-132). New York, NY: Oxford University Press.

Shune, S. \& Duff, M. C. (2014). Verbal play as a discourse resource in the social interactions of older and younger communication pairs. Journal of Interactional Research in Communication Disorders, 5(2), 193-216.

Smith, G. E., Housen, P., Yaffe, K., Ruff, R., Kennison, R. F., Mahncke, H. W., et al. (2009). A cognitive training program based on principles of brain plasticity: Results from the Improvement in Memory with Plasticity-based Adaptive Cognitive Training (IMPACT) study. Journal of the American Geriatrics Society, 57(4), 594-603.

Spector, A., Woods, B., \& Orrell, M. (2008). Cognitive stimulation for the treatment of Alzheimer's disease. Expert Review of Neurotherapeutics, 8(5), 751-757.

Stern, Y. (2002). What is cognitive reserve? Theory and research application of the reserve concept. Journal of the International Neuropsychological Society, 8(3), 448-460.

Stern, Y. (2009). Cognitive reserve. Neuropsychologia, 47(10), 2015-2028.
Stine-Morrow, E. A., Payne, B. R., Roberts, B. W., Kramer, A. F., Morrow, D. G., Payne, L., et al. (2014). Training versus engagement as paths to cognitive enrichment with aging. Psychology and Aging, 29(4), 891-906.

Suzuki, H., Kuraoka, M., Yasunaga, M., Nonaka, K., Sakurai, R., Takeuchi, R., et al. (2014). Cognitive intervention through a training program for picture book reading in community-dwelling older adults: A randomized controlled trial. BMC Geriatrics, 14, 122.

Torbeyns, T., Bailey, S., Bos, I., \& Meeusen, R. (2014). Active workstations to fight sedentary behaviour. Sports Medicine, 44(9), 1261-1273.

Uchida, S. \& Kawashima, R. (2008). Reading and solving arithmetic problems improves cognitive functions of normal aged people: A randomized controlled study. Age (Dordr), 30(1), 21-29.

Ukawa, S., Satoh, H., Yuasa, M., Ikeno, T., Kawabata, T., Araki, A., et al. (2012). A randomized controlled trial of a functioning improvement tool home-visit program and its effect on cognitive function in older persons. International Journal of Geriatric Psychiatry, 27(6), 557-564.

Verghese, J., Lipton, R. B., Katz, M. J., Hall, C. B., Derby, C. A., Kuslansky, G., et al. (2003). Leisure activities and the risk of dementia in the elderly. The New England Journal of Medicine, 348(25), 2508-2516.

Vohn, R. (2008). Functional networks of within-and cross-modal divided attention (unpublished doctoral dissertation). RWTH Aachen University, Aachen.

Wallin, A., Kettunen, P., Johansson, P. M., Jonsdottir, I. H., Nilsson, C., N ilsson, M., et al. (2018). Cognitive medicine-a new approach in health care science. BMC Psychiatry, 18(1), 42.

Willis, S. L. \& Caskie, G. I. (2013). Reasoning training in the ACTIVE study: How much is needed and who benefits? Journal of Aging and Health, 25(8 suppl), 43S-64S.

Wilson, R. S., Mendes De Leon, C. F., Barnes, L. L., Schneider, J. A., Bienias, J. L., Evans, D. A., et al. (2002). Participation in cognitively stimulating activities and risk of incident Alzheimer disease. Journal of the American Medical Association, 287(6), 742-748.

Zehnder, F., Martin, M., Altgassen, M., \& Clare, L. (2009). Memory training effects in old age as markers of plasticity: A meta-analysis. Restorative Neurology and Neuroscience, 27(5), 507-520. 